\title{
Influencia de la fusión espinal en la orientación del implante acetabular
}

Pablo D. López, Luis D. E. Orosco Falcone, Santiago L. Iglesias, Ignacio J. Pioli, José M. Gómez, Bartolomé L. Allende

Servicio de Ortopedia y Traumatología, Sanatorio Allende, Córdoba, Argentina

\begin{abstract}
RESUMEN
Introducción: Las enfermedades degenerativas de la cadera y la columna vertebral son causas comunes de discapacidad y dolor y los síntomas suelen superponerse. Cuando algún parámetro se altera, otro debe modificarse para evitar el choque femoroacetabular y una posible luxación. Se piensa que la fijación lumbar afectaría la adaptación de la unidad espino-pélvica en las diferentes posturas. El objetivo de este estudio fue analizar el comportamiento espino-pélvico en pacientes con artroplastia total de cadera y artrodesis lumbar. Materiales y Métodos: Se realizó un estudio no aleatorizado, retrospectivo, de casos y controles en pacientes con artroplastia total de cadera evaluados con radiografía lumbopélvica de frente y de perfil en posición erecta y en sedestación, divididos en dos grupos: con artrodesis lumbar o sin ella. Se midieron parámetros espino-pélvicos y femoroacetabulares. Resultados: La muestra tenía 50 pacientes: 25 en cada grupo. Quince tenían artroplastia total de cadera bilateral y el nivel de fijación lumbar más frecuente era L5-S1. No hubo diferencia estadísticamente significativa en la edad y el sexo entre ambos grupos. Los pacientes con artrodesis lumbar necesitaron más flexión de cadera para sentarse, sin un aumento significativo asociado en la tasa de luxación. Conclusiones: La composición ideal de los componentes aún es difícil de alcanzar. La reconsideración de las "zonas seguras" de los componentes ha comenzado a alejarse de los valores del plano coronal de Lewinnek. Se ha propuesto un nuevo enfoque en las zonas seguras del plano sagital más apropiadas y precisas en pacientes seleccionados con enfermedad espino-pélvica grave.
\end{abstract}

Palabras clave: Movilidad espino-pélvica; luxación, fijación lumbar.

Nivel de Evidencia: III

\section{Influence of Spinal Fusion on Acetabular Implant Orientation}

\section{ABSTRACT}

Introduction: Degenerative diseases of the hip and spine are common causes of disability and pain, and the symptoms usually overlap. When a parameter is altered, another one should be modified to avoid femoroacetabular impingement and a potential dislocation. It is believed that lumbar fixation would affect the adaptation of the spinopelvic unit in different postures. This article aims to analyze the spinopelvic behavior in patients with Total Hip Arthroplasty (THA) and lumbar arthrodesis. Materials and Methods: A non-randomized retrospective study of cases and controls was carried out in patients with THA, who were assessed using anterior and lateral X-ray views in functional sitting and standing postures, divided into two groups depending on the presence or absence of lumbar arthrodesis. Spinopelvic parameters as well as femoroacetabular parameters were measured. Results: $\mathrm{A}$ sample of 50 patients was selected, 25 in each group. In total, 15 patients had bilateral THA, and the most common level of lumbar fixation was L5-S1. There was no statistically significant difference in gender and age between both groups. Lumbar arthrodesis patients required more hip flexion to sit, without being associated with a significant increase in the rate of dislocation. Conclusion: The ideal composition of the components is still difficult to achieve. The review of the "safe zones" of the components has started to depart from the values of the body plane proposed by Lewinnek. A new approach has been proposed to the safe zones of the sagittal plane, which are more appropriate and accurate in selected patients with severe spinopelvic pathology. Keywords: Spinopelvic mobility; dislocation; lumbar fixation.

Level of Evidence: III Recibido el 16-1-2021. Aceptado luego de la evaluación el 28-4-2021 • Dr. PABLO D. LÓPEZ • pablopez1292@ gmail.com ID https://orcid.org/0000-0001-9722-1317
Cómo citar este artículo: López PD, Orosco Falcone LDE, Iglesias SL, Pioli IJ, Gómez JM, Allende BL. Influencia de la fusión espinal en la orientación del implante acetabular. Rev Asoc Argent Ortop Traumatol 2021;86(4):463-474. https://doi.org/10.15417/issn. 1852-7434.2021.86.4.1302 


\section{INTRODUCCIÓN}

Las enfermedades degenerativas de la cadera y la columna vertebral son causas comunes de discapacidad y dolor. El diagnóstico y el tratamiento de las afecciones relacionadas con la cadera y la columna vertebral son un desafío debido a la superposición de síntomas. La artroplastia total de cadera (ATC) y la fusión de la columna lumbar pueden aliviar eficazmente el dolor y mejorar la función en pacientes adecuadamente seleccionados con un cuadro degenerativo y, como resultado, los volúmenes de estas operaciones están aumentando. No es raro tener una fusión espinal y una ATC..$^{-3}$

En la ATC, la colocación precisa de la copa acetabular es esencial para lograr una amplitud de movimiento libre y estable. Durante décadas, los cirujanos ortopédicos se han basado en la "zona segura" para la colocación del implante acetabular, descrita por Lewinnek y cols. $\left(40^{\circ} \pm 10^{\circ} \text { de abducción y } 15^{\circ} \pm 10^{\circ} \text { de anteversión }\right)^{4}$ para reducir la inestabilidad de la prótesis, pero actualmente, debido a nuevos conocimientos del papel que juega la unidad espino-pélvica en la orientación acetabular, este concepto está cambiando. ${ }^{5}$ Además, la orientación acetabular es un parámetro dinámico que puede verse afectado por fuerzas que pueden originarse desde la parte superior de la articulación de la cadera (p. ej., cambio en la alineación espinal), en la cadera (p. ej., debilidad muscular o degeneración avanzada de cadera) o debajo de la cadera (p. ej., discrepancia entre las extremidades). ${ }^{6}$

Estudios recientes hallaron una alta tasa de luxaciones de las prótesis que se encontraban dentro de la "zona segura" de Lewinnek, por lo que sugieren que otros factores pueden jugar un papel en la inestabilidad de la cadera. ${ }^{5,7}$

La fusión espinal puede alterar la adaptación de la unión espino-pélvica, y puede dar como resultado una anteversión y una inclinación del implante acetabular inferior a la óptima en sedestación y bipedestación y, a su vez, dislocación o subluxación. ${ }^{8}$

\section{Movilidad espino-pélvica}

Cada persona se caracteriza por un parámetro "morfológico": el ángulo de incidencia pélvica (pelvic inciden$c e, \mathrm{PI}$ ), que representa esquemáticamente el grosor pélvico. La adaptación de otros factores funcionales, como la inclinación pélvica (pelvic tilt, PT) y los parámetros espinales [inclinación sacra (sacral slope, SS), lordosis lumbar (LL) y cifosis torácica] permiten posicionar el centro de gravedad del tronco específicamente para que esté apoyado por las cabezas femorales relativas a la base pélvica, para mantener el equilibrio con solo un mínimo esfuerzo muscular. Matemáticamente todos los parámetros pélvicos son unidos mediante la siguiente fórmula: $\mathrm{PI}=\mathrm{PT}+\mathrm{SS}$.

La pelvis se mueve, girando alrededor del eje bicoxofemoral, lo que lleva a la inclinación anterior (donde la porción superior de la pelvis se inclina hacia adelante) y la inclinación posterior (la parte superior de la pelvis se inclina hacia atrás). Las variaciones en los ángulos de la pendiente sacra determinan el rango de esta PT. ${ }^{9}$

La posición erecta corresponde a una inclinación hacia delante de la pelvis como un todo. En esta situación, la placa superior de $\mathrm{S} 1$, vista lateralmente, forma un ángulo de aproximadamente $35^{\circ}$ a $45^{\circ}$ con la horizontal..$^{9-11}$ Algunas personas tienen un pequeño ángulo de SS en posición erecta: hablamos entonces de PT posterior (o retroversión pélvica o extensión pélvica), y el sacro visto en una imagen lateral parece más vertical que lo normal. Por el contrario, otras personas tienen un sacro muy horizontal en posición erecta con un ángulo de SS, a veces, mucho mayor que $50^{\circ}$ (PT anterior o anteversión pélvica o flexión pélvica).

En una posición sentada, el fenómeno se invierte. La pelvis se inclina hacia atrás a medida que avanza hacia la sedestación. La SS disminuye, a valores de $20^{\circ}$ a $25^{\circ}$ en promedio. ${ }^{9,10,12}$ Esta pendiente puede ser ligeramente positiva $\left(5^{\circ}-10^{\circ}\right)$ o incluso negativa. Como función de la altura del asiento, la morfología del individuo o cualquier enfermedad espinal asociada, observamos la inclinación posterior de la pelvis (retroversión pélvica o extensión pélvica) más o menos acentuada con un sacro más o menos vertical.

Esto es particularmente confuso para los cirujanos de artroplastia que generalmente se refieren a la retroversión en relación con la copa acetabular, que es el movimiento opuesto. Por ejemplo, con la PT posterior o la retroversión pélvica, la posición funcional de la copa acetabular se vuelve más anteversa.

Estas modificaciones en los parámetros espino-pélvicos llevan a los cambios correspondientes en la orientación acetabular [inclinación acetabular (acetabular inclination, AI) y anteversión acetabular (AA)]. El valor combinado de estas dos medidas de la orientación acetabular es el ángulo de anteinclinación (AAI). En posición erecta, el valor de la SS es alto y el ángulo de la AA es pequeño. Inversamente, en sedestación, la SS disminuye y la AA aumenta. Radiográficamente tanto en posición anteroposterior como lateral, el implante acetabular aparece más vertical en sedestación que en posición erecta. 
Por cada $1^{\circ}$ de PT posterior, la AA funcional aumentará $0,7^{\circ}-0,8^{\circ}$. El cambio en la AI funcional es menos significativo y no lineal, según el grado de PT. En general, la PT posterior mayor aparecerá más como una vista de salida, mientras que una PT anterior mayor aparecerá más como una vista de entrada en la radiografía anteroposterior de pelvis.

El objetivo de este estudio fue analizar el comportamiento de la ATC en pacientes con artrodesis lumbar y sin ella.

\section{MATERIALES Y MÉTODOS}

Se llevó a cabo un estudio no aleatorizado, retrospectivo, de casos y controles de pacientes con ATC que fueron evaluados con radiografías de frente y de perfil en posición funcional erecta y en sedestación.

El estudio se realizó en conjunto con los profesionales del Servicio de Diagnóstico por Imágenes, a quienes se les entregó un protocolo en el que se explicaban los criterios de inclusión y exclusión, y las normativas para obtener las radiografías.

En las radiografías, se debían visualizar, con claridad, ambas cabezas femorales o prótesis con tercio proximal de fémur, sínfisis púbica en perfil, columna vertebral desde el platillo superior de L1, platillo superior de S1.

Se incluyeron pacientes de nuestro Servicio de Ortopedia y Traumatología sometidos a una ATC. Se obtuvieron registros de las historias clínicas para clasificarlos en dos grupos, según si tenían o no patología espinal: grupo A (ATC más fijación lumbar) y grupo B o de control (ATC sin fijación lumbar).

Se midieron los parámetros espino-pélvicos LL, PT, PI, SS, el plano pélvico anterior (anterior pelvic plane, APP), y los parámetros femoroacetabulares AAI, ángulo pélvico femoral (pelvic femoral angle, PFA). Las mediciones se realizaron en bipedestación y sedestación mediante el programa SurgiMap ${ }^{13}$ para Windows (Figuras 1 y 2).

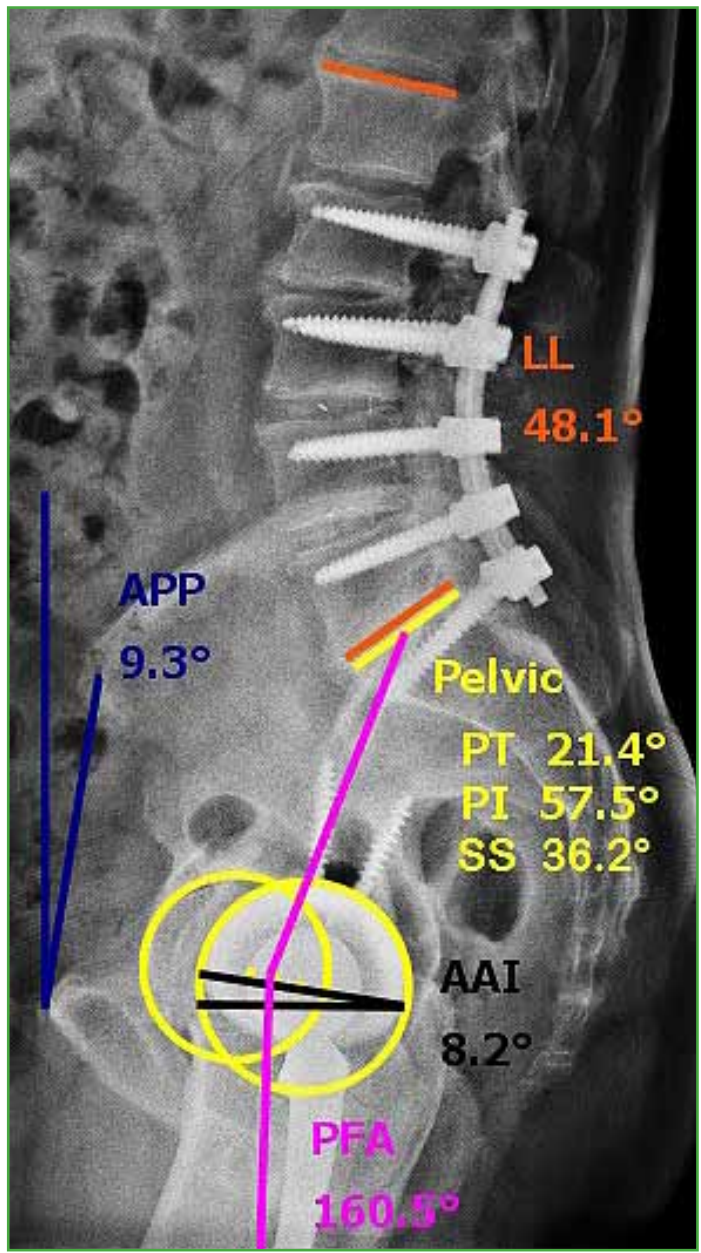

Figura 1. Medición en bipedestación de un paciente con artrodesis de cuatro niveles. PI = incidencia pélvica, $\mathrm{PT}=$ inclinación pélvica, $\mathrm{SS}=$ inclinación sacra, $\mathrm{LL}=$ lordosis lumbar, $\mathrm{AAI}=$ anteinclinación acetabular, $\mathrm{PFA}=$ ángulo pélvico femoral, APP = plano pélvico anterior. 


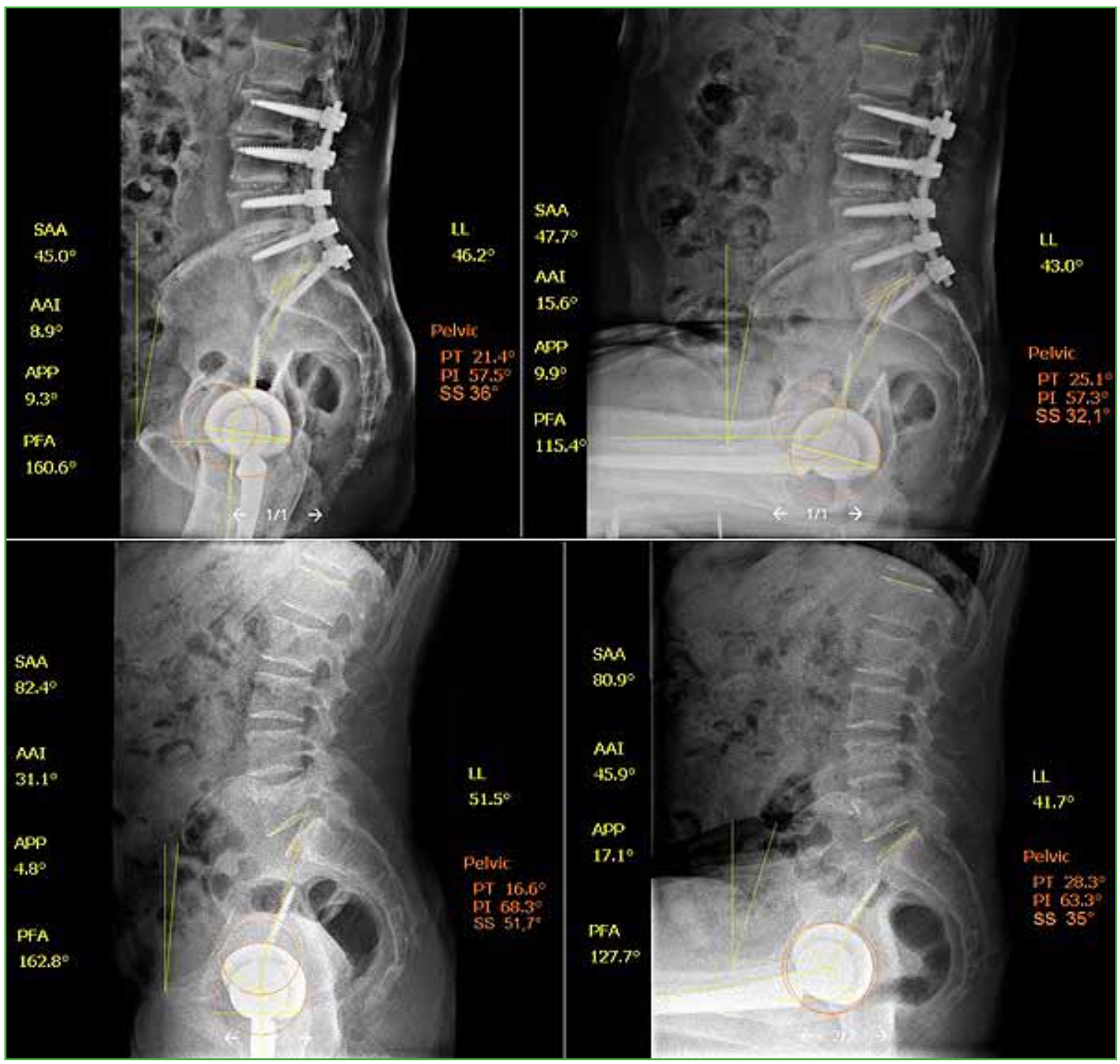

Figura 2. Mediciones realizadas a los pacientes del grupo A (imagen superior) y del grupo B (imagen inferior).

PI: es el ángulo que se forma entre una línea trazada desde el centro de la cabeza femoral hasta el punto medio en el platillo superior de S1 y una segunda línea perpendicular a este último punto.

PT: es el ángulo obtenido entre una línea que va desde el centro de la cabeza femoral hasta un punto medio localizado en el platillo superior de S1 y una segunda línea vertical de referencia a $0^{\circ}$.

SS: es un ángulo formado entre una línea que se traza paralela al eje del platillo superior de S1 y una segunda línea de referencia horizontal a $0^{\circ}$.

APP: representa la PT, pero medido en el perfil como el ángulo que se forma por dos líneas, una desde la sínfisis púbica a la espina ilíaca anterosuperior y otra vertical a $0^{\circ}$ desde la sínfisis púbica.

LL: es el grado de lordosis de la columna lumbar medida desde el platillo superior de L1 hasta el platillo superior de S1.

AAI: medido en el perfil con una línea que conecta el borde acetabular desde la porción más anterior a la más posterior con otra línea de referencia a $0^{\circ}$.

PFA: es el ángulo que representa la flexión de la cadera y se mide con una línea que conecta el centro de la cabeza femoral con la diáfisis femoral y otra línea desde el centro de la cabeza femoral hacia el punto medio del platillo superior de S1. 


\section{Análisis estadístico}

Se realizó un análisis descriptivo global usando medidas resumen y gráficos exploratorios, para cada característica y de manera bivariada. Para estudiar la relación entre las variables cualitativas, se empleó la prueba de la ji al cuadrado de Pearson.

Para estudiar las diferencias entre grupos de variables cuantitativas, se utilizó la prueba t de Student para muestras independientes. Por último, se utilizó el programa SPSS para Windows v. 22 para los análisis estadísticos, y el programa Excel para confeccionar los gráficos y las tablas. El nivel de significación utilizado en todos los casos fue 0,05 .

\section{Aspectos éticos}

Todos los pacientes dieron el consentimiento informado verbal y escrito para participar de la investigación. El protocolo de investigación fue aprobado por el Comité de Ética Institucional y cumple con la Declaración de Helsinki y la Declaración de Buenas Prácticas Clínicas de ANMAT. También cumple con la Ley de la Provincia de Córdoba $\mathrm{N}^{\circ} 9694$ y la Ley Nacional Argentina de protección de datos personales $\mathrm{N}^{\circ} 25.326$.

\section{RESULTADOS}

Se obtuvo una muestra de 50 pacientes, 25 para cada grupo. En el grupo A, el 68\% eran mujeres, con una edad promedio (desviación estándar) de 70 años (9.62) entre ambos sexos. El grupo B estaba formado por un $56 \%$ de mujeres, con una edad promedio (desviación estándar) de 68 años (11.03) entre ambos sexos. No había una diferencia estadísticamente significativa entre ambos grupos en cuanto a la edad $(p=0,566)$ y el sexo $(\mathrm{p}=0,297)$.

Ocho pacientes del grupo A y siete del grupo B tenían una ATC bilateral (en total 15 pacientes [80\% mujeres]). En el grupo A, el nivel de fijación lumbar más frecuente fue L5-S1 (7 pacientes, 28\%) (Tabla 1).

Tabla 1. Frecuencia de niveles de artrodesis en el grupo $\mathrm{A}^{*}$

\begin{tabular}{|c|c|c|c|c|c|}
\hline Artrodesis & $\begin{array}{c}\text { Frecuencia } \\
\text { absoluta }\end{array}$ & Total & Cociente & $\begin{array}{c}\text { Frecuencia } \\
\text { relativa }\end{array}$ & $\%$ \\
\hline L5-S1 & 7 & 25 & $7 / 25$ & 0,28 & $28 \%$ \\
\hline L3-L4-L5 & 3 & 25 & $3 / 25$ & 0,12 & $12 \%$ \\
\hline L4-L5-S1 & 3 & 25 & $3 / 25$ & 0,12 & $12 \%$ \\
\hline L2-L5 & 2 & 25 & $2 / 25$ & 0,08 & $8 \%$ \\
\hline L4-L5 & 2 & 25 & $2 / 25$ & 0,08 & $8 \%$ \\
\hline L4-S1 & 2 & 25 & $2 / 25$ & 0,08 & $8 \%$ \\
\hline L2-S1 & 1 & 25 & $1 / 25$ & 0,04 & $4 \%$ \\
\hline L3-L4-L5-S1 & 1 & 25 & $1 / 25$ & 0,04 & $4 \%$ \\
\hline L3-S1 & 1 & 25 & $1 / 25$ & 0,04 & $4 \%$ \\
\hline T10-S1 & 1 & 25 & $1 / 25$ & 0,04 & $4 \%$ \\
\hline $\mathrm{T} 2-\mathrm{S} 1$ & 1 & 25 & $1 / 25$ & 0,04 & $4 \%$ \\
\hline T6-S1 & 1 & 25 & $1 / 25$ & 0,04 & $4 \%$ \\
\hline
\end{tabular}

"El nivel L5-S1 es el más frecuente. 
Al calcular los porcentajes de pacientes con luxación, fue superior en el grupo de artrodesis que en el grupo control: 12\% vs. 4\% (Figura 3); sin embargo, la diferencia no fue significativa.

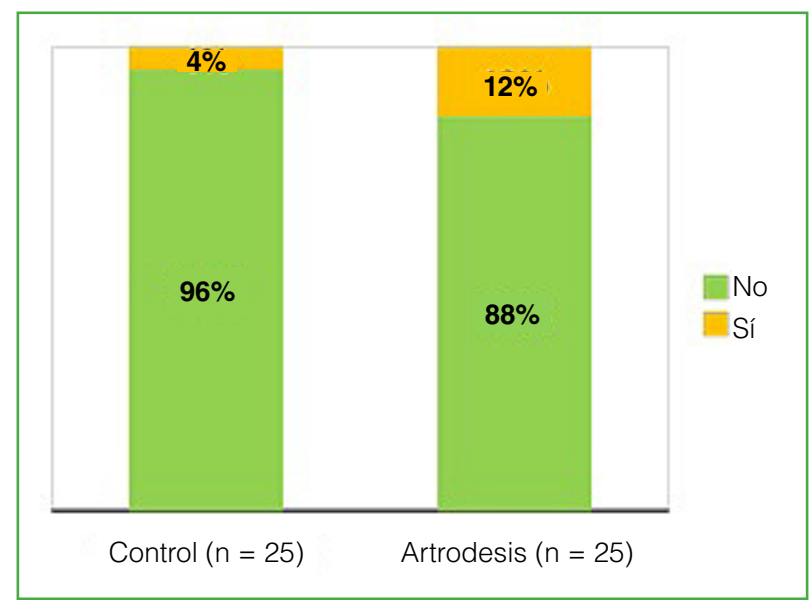

Figura 3. Luxación de la artroplastia total de cadera según los grupos de pacientes $(\mathrm{p}=0,2971)$.

\section{Parámetros espino-pélvicos (Tabla 2)}

Los pacientes del grupo de control tenían un mayor promedio de LL (posición erecta) y un menor promedio de LL (sedestación), pero la diferencia entre los grupos no fue estadísticamente significativa ( $p>0,05)$. Asimismo, estos presentaban un menor promedio de PT (posición erecta), y la diferencia entre los grupos fue estadísticamente significativa $(p=0,0269)$. En cambio, no hubo diferencias estadísticamente significativas entre los grupos con respecto a la PT (sedestación).

En cuanto a la PI, hubo una diferencia de medias entre la posición erecta y la sedestación de $1,21^{\circ}$ en el grupo A y de $3^{\circ}$ en el grupo $B$, sin diferencias estadísticamente significativas entre ambos ( $p>0,05$ ).

Los pacientes del grupo B tenían un mayor promedio de SS (posición erecta), y la diferencia entre los grupos fue estadísticamente significativa $(\mathrm{p}=0,0002)$. La SS se modificó, en promedio, $8,3^{\circ}$ en los pacientes con artrodesis lumbar y $20^{\circ}$ en aquellos sin artrodesis lumbar. Esta diferencia de medias entre los grupos fue estadísticamente significativa $(\mathrm{p}=0,001)$ (Figura 4$)$.

Los pacientes del grupo sin artrodesis tenían un menor promedio de APP en posición erecta y sedestación, pero la diferencia entre los grupos no fue estadísticamente significativa $(p>0,05)$.

\section{Parámetros femoroacetabulares}

Los pacientes del grupo de control presentaban un mayor promedio de AAI (posición erecta y sedestación), pero la diferencia entre los grupos no fue estadísticamente significativa $(\mathrm{p}>0,05)$.

Los pacientes con artrodesis tenían un aumento promedio de $11^{\circ}$ en el AAI al pasar de la posición erecta a la sedestación y, en aquellos sin artrodesis lumbar, este valor aumentó $17,30^{\circ}$, sin diferencia estadísticamente significativa (Figura 5).

Los pacientes del grupo de artrodesis presentaron un menor promedio de PFA (bipedestación), y la diferencia entre los grupos fue estadísticamente significativa $(p=0,0049)$. En cambio, no hubo diferencias estadísticamente significativas entre los grupos con respecto al PFA (sedestación). Al enfrentar ambos grupos, los pacientes en general del grupo con artrodesis lumbar flexionaron $10,50^{\circ}$ más la cadera que aquellos sin artrodesis al pasar de la posición erecta a la sedestación ( $\mathrm{p}>0,05$ ) (Figura 6). 
Tabla 2. Estadística general de los parámetros espino-pélvicos según los grupos

\begin{tabular}{|c|c|c|c|c|}
\hline Variables & Posición & $\begin{array}{l}\text { Artrodesis } \\
\quad(n=25)\end{array}$ & $\begin{array}{l}\text { Control } \\
(n=25)\end{array}$ & p* \\
\hline \multirow[t]{3}{*}{ PT } & Erecta & $24,6 \pm 10,7$ & $17,8 \pm 9,8$ & 0,0269 \\
\hline & Sedestación & $32,0 \pm 13,0$ & $35,5 \pm 15,4$ & 0,3516 \\
\hline & $\mathbf{p}^{* *}$ & 0,0001 & 0,0001 & --- \\
\hline \multirow[t]{3}{*}{ PI } & Erecta & $58,7 \pm 15,7$ & $64,6 \pm 14,0$ & 0,1838 \\
\hline & Sedestación & $57,5 \pm 13,5$ & $61,6 \pm 11,8$ & 0,1870 \\
\hline & $\mathrm{p}^{* *}$ & 0,5073 & 0,0969 & --- \\
\hline \multirow[t]{3}{*}{ SS } & Erecta & $34,1 \pm 11,7$ & $47,0 \pm 10,7$ & 0,0002 \\
\hline & Sedestación & $25,8 \pm 10,5$ & $27,0 \pm 13,2$ & 0,7196 \\
\hline & $\mathrm{p}^{* *}$ & 0,0001 & 0,0001 & --- \\
\hline \multirow[t]{3}{*}{ LL } & Erecta & $41,3 \pm 17,1$ & $47,4 \pm 12,4$ & 0,2142 \\
\hline & Sedestación & $34,9 \pm 14,6$ & $33,7 \pm 14,9$ & 0,4668 \\
\hline & $\mathrm{p}^{* *}$ & 0,0001 & 0,0017 & --- \\
\hline \multirow[t]{3}{*}{ SAA } & Erecta & $68,7 \pm 17,2$ & $79,2 \pm 14,3$ & 0,0226 \\
\hline & Sedestación & $70,7 \pm 15,1$ & $75,7 \pm 13,8$ & 0,1806 \\
\hline & $\mathrm{p}^{* *}$ & 0,3872 & 0,0530 & --- \\
\hline \multirow[t]{3}{*}{ AAI } & Erecta & $34,1 \pm 12,7$ & $32,7 \pm 9,0$ & 0,4550 \\
\hline & Sedestación & $45,1 \pm 11,7$ & $50,0 \pm 13,6$ & 0,1936 \\
\hline & $\mathrm{p}^{* *}$ & 0,0001 & 0,0001 & --- \\
\hline \multirow[t]{3}{*}{ APP } & Erecta & $15,3 \pm 33,4$ & $5,4 \pm 4,0$ & 0,1402 \\
\hline & Sedestación & $14,8 \pm 10,1$ & $16,4 \pm 11,2$ & 0,6836 \\
\hline & $\mathrm{p}^{* *}$ & 0,9408 & 0,0001 & --- \\
\hline \multirow[t]{3}{*}{ PFA } & Erecta & $168,4 \pm 9,6$ & $159,3 \pm 11,2$ & 0,0049 \\
\hline & Sedestación & $131,8 \pm 17,0$ & $133,2 \pm 17,0$ & 0,6554 \\
\hline & $\mathrm{p}^{* *}$ & 0,0001 & 0,0001 & --- \\
\hline
\end{tabular}

${ }^{*}$ Prueba T para muestras independientes. ${ }^{* *}$ Prueba $\mathrm{T}$ para muestras apareadas.

$\mathrm{PT}=$ pelvic tilt (inclinación pélvica), PI = pelvic incidence (incidencia pélvica), SS = sacral slope (inclinación sacra), LL = lordosis lumbar,

$\mathrm{SAA}=$ sacroacetabular angle (ángulo sacroacetabular), AAI = ángulo de anteinclinación, APP = anterior pelvic plane (plano pélvico anterior),

PFA = pelvic femoral angle (ángulo pélvico femoral). 


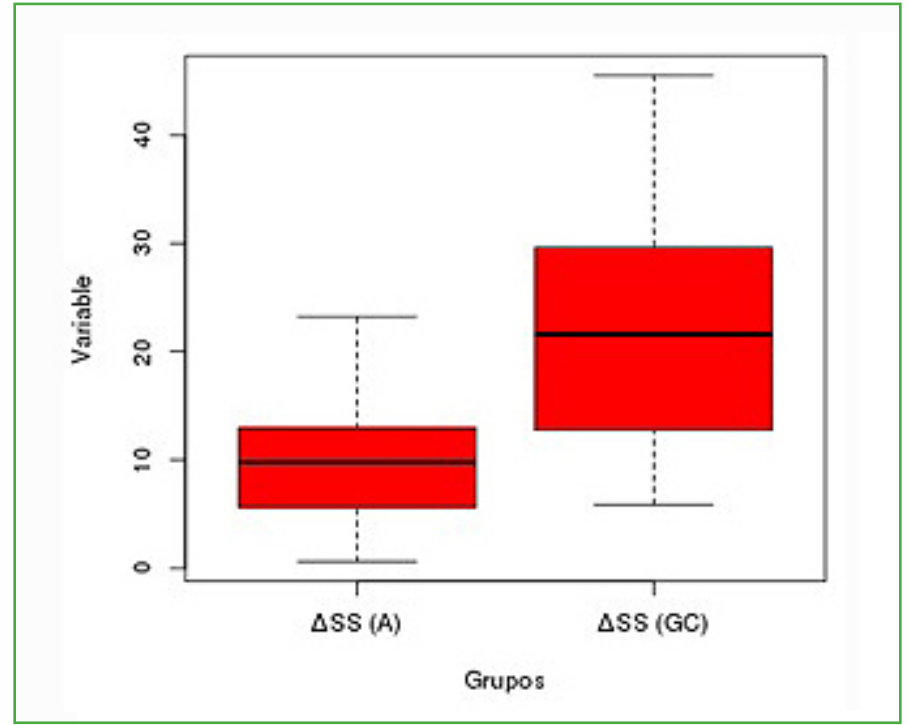

Figura 4. Diferencia de medias de la inclinación sacra (SS) entre los grupos con artrodesis (A) y el grupo de control (GC) (estadísticamente significativa, $\mathrm{p}=0,001)$.

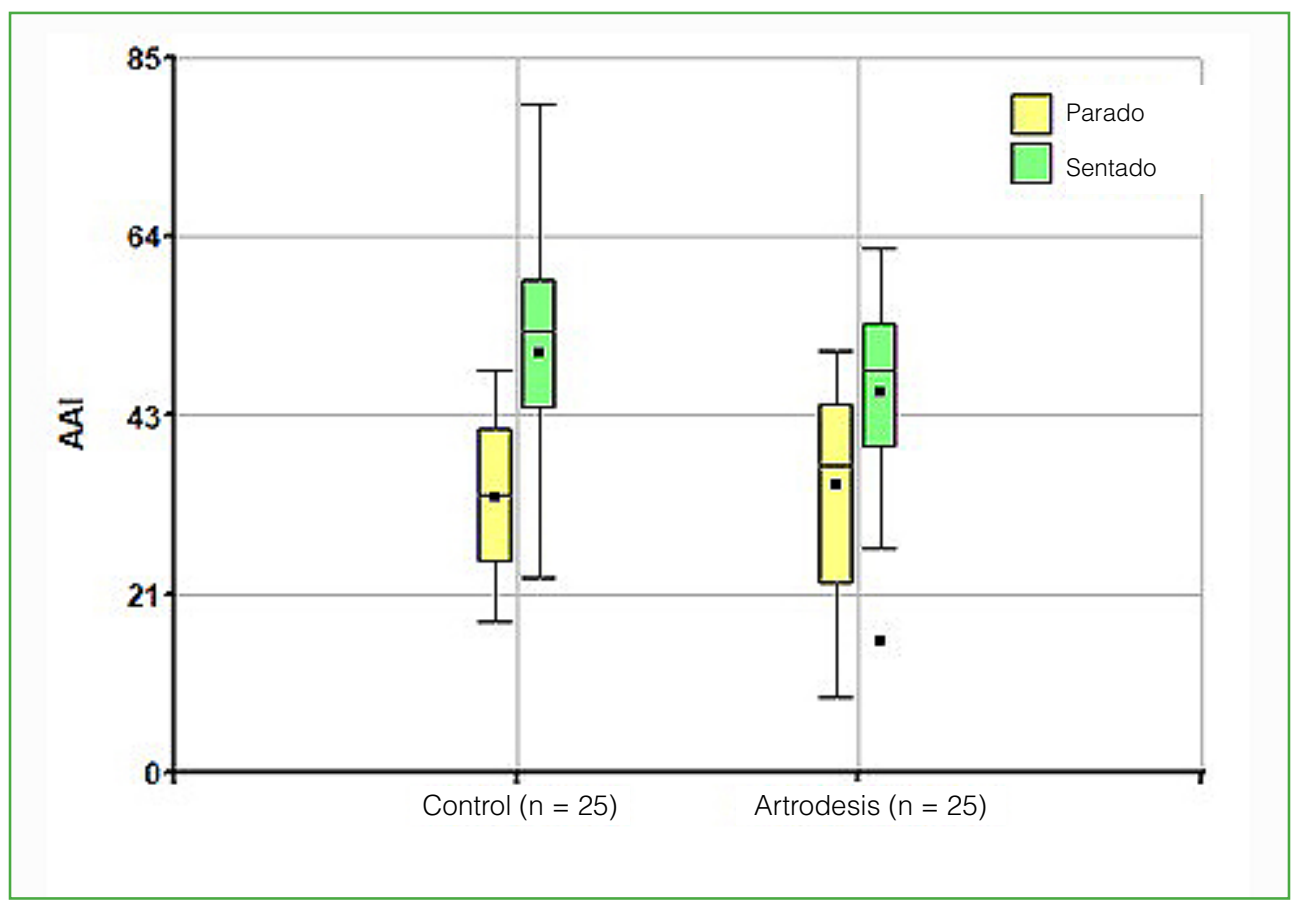

Figura 5. Diagrama de cajas de la anteinclinación acetabular (AAI) (bipedestación y sedestación) según los grupos de pacientes. 


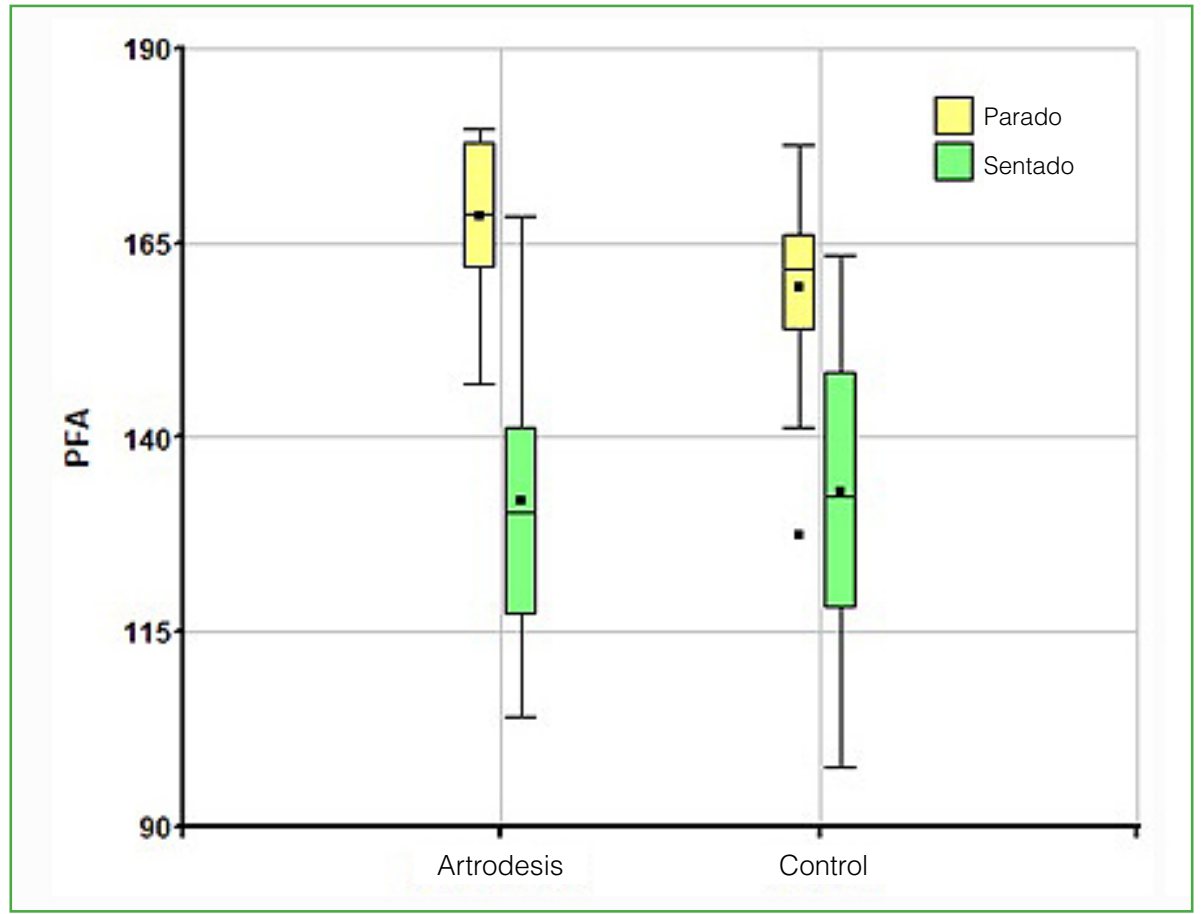

Figura 6. Diagrama de cajas del ángulo pélvico femoral (PFA) (bipedestación y sedestación) según los grupos de pacientes.

\section{DISCUSIÓN}

La movilidad espino-pélvica puede ser confusa. La mayoría de los cirujanos que realizan artroplastias se centran en el posicionamiento del componente acetabular de acuerdo con la posición estática de la pelvis. Sin embargo, en su lugar, debe usarse la posición funcional. Esto explicará la interacción dinámica entre la columna vertebral, la pelvis y la cadera. En los últimos años, han aumentado las investigaciones sobre la influencia de la movilidad espino-pélvica, y la inclinación y anteversión del componente acetabular para la ATC.,10

La mayoría de los pacientes sometidos a ATC tendrán un movimiento espino-pélvico normal $\left(\Delta \mathrm{SS} 20^{\circ}-40^{\circ}\right.$ de posición erecta a sedestación) y no tendrán un desequilibrio sagital clínicamente significativo (PT posición erecta $\pm 10^{\circ}$ ). Además, Stefl y cols. ${ }^{14}$ comunicaron que el $16 \%$ de los pacientes con anomalías espino-pélvicas preoperatorias recuperaron el movimiento espino-pélvico normal después de la ATC, presumiblemente debido a la liberación de contracturas en flexión de cadera. Como resultado, para la mayoría de los pacientes, la colocación del componente acetabular en el plano coronal estándar (zona segura de Lewinnek) ha logrado excelentes resultados durante tantos años. Incluso la ATC en pacientes con anormalidades espino-pélvicas menores se ha mantenido históricamente a salvo de la luxación de la prótesis, porque los cirujanos tienden a apuntar a ángulos de inclinación y anteversión acetabulares estrechos de $30^{\circ}-45^{\circ}$ y $15^{\circ}-20^{\circ}$, respectivamente. ${ }^{15}$ Sin embargo, debe reconocerse que existe un espectro de inestabilidad que incluye dolor por pinzamiento sin dislocación franca. Para los pacientes de alto riesgo con movilidad espino-pélvica patológica, varios autores han descrito esquemas de clasificación y han proporcionado posibles soluciones.

Dorr y cols. ${ }^{16}$ clasificaron a la movilidad espino-pélvica en tres categorías sobre la base de la diferencia en la SS entre la posición erecta y la sedestación. La clasificación se divide en: normal $\left(20^{\circ}-40^{\circ}\right)$, hipermóvil $\left(>40^{\circ}\right)$ y rígida $\left(<20^{\circ}\right)$. Señalaron que, mientras que el movimiento espino-pélvico normal e hipermóvil no tenía casi riesgo de pinzamiento y dislocación, el movimiento espino-pélvico rígido tenía un mayor riesgo de dislocación debido a un desequilibrio. En nuestra serie, los pacientes con artrodesis lumbar presentaron un promedio de $8,30^{\circ}$ (rígida) y los pacientes sin artrodesis lumbar, un promedio de $20^{\circ}$ (normal).

Stefl y cols., y Kanawade y cols. ${ }^{14,17}$ sugirieron que las pelvis con movilidad normal e hipermóviles tienden a tolerar una gran variabilidad en la posición de la copa, el choque y la inestabilidad debido a la biomecánica 
espino-pélvica normal. Durante la colocación de la copa, se recomienda mantener la técnica quirúrgica normal y la implantación de la zona de seguridad de la copa regular de $15^{\circ} \pm 10^{\circ}$ de anteversión y $40^{\circ} \pm 10^{\circ}$ de inclinación. A su vez, recomendaron que las pelvis rígidas, que demuestran $<10^{\circ}$ de cambio de SS entre la posición erecta y la sedestación, se clasifiquen por la posición acetabular en la que se encuentran. La posición "erecta" o de inclinación anterior indica un acetábulo más horizontal y un choque anterior; por lo tanto, un riesgo de dislocación posterior. La posición "sentada" o de inclinación posterior indica un posible choque posterior e inestabilidad anterior. Estas clasificaciones ayudan a guiar al cirujano a la ubicación ideal para el implante de la copa. Estas clasificaciones pueden ser útiles como categorías generales, pero el grado de rigidez y desequilibrio sagital debe determinarse caso por caso. Además, a medida que los pacientes envejecen o se someten a procedimientos quirúrgicos, pueden pasar de una categoría a otra. La enfermedad de la columna es progresiva y la pérdida de movilidad espino-pélvica y el equilibrio sagital pueden ser responsables de luxaciones tardías.

Los objetivos de la posición de la copa en una clase rígida espino-pélvica son $45^{\circ}-50^{\circ}$ de inclinación $\left(50^{\circ}\right.$ en pacientes ancianos y $45^{\circ}$ en los más jóvenes) y $20^{\circ}-25^{\circ}$ de anteversión. Se considera la prótesis de doble movilidad si los valores del AAI de un paciente cambian $<5^{\circ}$ entre sentarse y pararse, lo que significa que el acetábulo no se adapta al movimiento espino-pélvico y está en riesgo de luxación.

Una revisión de los datos de Medicare halló un aumento del 293\% de pacientes con fusión lumbar sometidos a ATC durante un período de 12 años. ${ }^{18}$ La prevalencia de enfermedad degenerativa de la columna lumbar en pacientes sometidos a ATC primaria por osteoartritis de cadera fue aproximadamente del $40 \% .{ }^{14} \mathrm{El}$ efecto de la enfermedad de la columna vertebral en la ATC se ha centrado, en gran medida, en el riesgo de luxación. Según grandes estudios multicéntricos, la inestabilidad posquirúrgica de la cadera es del $2 \%$ al 4\%. ${ }^{19,20}$ Sin embargo, los estudios contemporáneos que se centran en ATC en pacientes con enfermedad degenerativa de la columna vertebral o una fusión lumbar de segmento largo han detectado un riesgo de luxación del 8\% al 18\%.5.18

Bedard y cols..$^{21}$ observaron que los pacientes con fusión espino-pélvica y ATC tenían una tasa de luxación del $20 \%$ en su institución y del 8,3\% en la base de datos nacional de los Estados Unidos. Llegaron a la conclusión de que se trataba de una tasa alarmantemente alta en comparación con las tasas de control del 2,9\%.

Perfetti y cols. ${ }^{22}$ notaron una tasa de luxación siete veces mayor con una fusión vertebral previa y varios autores han comunicado una asociación positiva con el número de niveles fusionados y el grado de desequilibrio espinal. ${ }^{6,23}$

Malkani y cols. ${ }^{18}$ descubrieron que la fusión lumbar realizada dentro de los cinco años anteriores a la ATC era un factor de riesgo independiente para la luxación y corrobora los datos publicados previamente..$^{22,23}$

Existe un posible nuevo conjunto de riesgos en estos pacientes si la cirugía de fusión lumbar se realiza después de la ATC debido al reajuste de la PT posterior, la anteversión acetabular funcional y la rigidez espinal.

En nuestra serie, tuvimos tres luxaciones en el grupo de artrodesis (12\%) y un solo caso de luxación de la prótesis en el grupo de control (4\%); sin embargo, esta diferencia no fue significativa. No hubo diferencias estadísticamente significativas en los parámetros medidos para ese caso en comparación con el resto de los pacientes del mismo grupo. Por lo tanto, no podemos afirmar que esta luxación se debió a un desequilibrio espino-pélvico o acetabular.

Dorr y cols. ${ }^{24,25}$ clasificaron a los pacientes con dislocaciones de cadera por sus etiologías subyacentes. Si bien atribuyeron la mayoría a una causa identificable, el 17\% no tenía una etiología conocida. Esto puede sugerir otros factores de riesgo para la inestabilidad desconocidos en ese momento.

Como limitaciones de este estudio mencionamos la baja cantidad de pacientes y no contar con un adecuado método radiológico que nos permita disminuir las oblicuidades periféricas de las radiografías al tener que ampliar al máximo el colimador. Creemos que esto se puede mejorar en posteriores estudios y con una mejor comunicación entre técnicos radiólogos y médicos solicitantes.

\section{CONCLUSIONES}

La artrodesis lumbar reduce la movilidad espino-pélvica y, en consecuencia, la adaptación acetabular a los cambios de posición; los pacientes con artrodesis necesitan más flexión de cadera para sentarse, sin que este factor se asocie, de manera significativa, con la luxación de la prótesis. A medida que avanza nuestra apreciación y comprensión, esperamos poder identificar, con mayor exactitud, a los pacientes de alto riesgo (antecedentes patológicos de la columna y cadera). A su vez, un menor daño de los tejidos blandos también puede mitigar el riesgo de inestabilidad. Se necesitan más estudios a futuro para identificar y reducir esta zona segura sagital propuesta. No obstante, en algunos casos de movimiento patológico grave, pueden sugerirse implantes de articulación de doble movilidad, particularmente en cirugías de revisión. 
Conflicto de intereses: Los autores no declaran conflictos de intereses.

ORCID de L. D. E. Orosco Falcone: https://orcid.org/0000-0003-0988-305X ORCID de S. L. Iglesias: https://orcid.org/0000-0002-1823-0416 ORCID de I. J. Pioli: https://orcid.org/0000-0001-8697-1980
ORCID de J. M. Gómez: https://orcid.org/0000-0002-1162-2708 ORCID de B. L. Allende: https://orcid.org/0000-0003-2757-4381

\section{BIBLIOGRAFÍA}

1. Fogel GR, Esses SI. Hip spine syndrome: management of coexisting radiculopathy and arthritis of the lower extremity. Spine J 2003;3:238-41. https://doi.org/10.1016/s1529-9430(02)00453-9

2. McNamara MJ, Barrett KG, Christie MJ, Spengler DM. Lumbar spinal stenosis and lower extremity arthroplasty. $J$ Arthroplasty 1993;8:273-7. https://doi.org/10.1016/s0883-5403(06)80089-6

3. Saito J, Ohtori S, Kishida S, Nakamura J, Takeshita M, Shigemura T, et al. Difficulty of diagnosing the origin of lower leg pain in patients with both lumbar spinal stenosis and hip joint osteoarthritis. Spine (Phila Pa 1976) 2012;37: 2089-93. https://doi.org/10.1097/BRS.0b013e31825d213d

4. Lewinnek GE, Lewis JL, Tarr R, Compere CL, Zimmerman JR. Dislocations after total hip-replacement arthroplasties. J Bone Joint Surg Am 1978;60:217-20. PMID 641088

5. Abdel MP, von Roth P, Jennings MT, Hanssen AD, Pagnano MW. What safe zone? The vast majority of dislocated THAs are within the Lewinnek safe zone for acetabular component position. Clin Orthop Relat Res 2016;474:38691. https://doi.org/10.1007/s11999-015-4432-5

6. Sultan AA, Khlopas A, Piuzzi NS, Chughtai M, Sodhi N, Mont MA. The impact of spino-pelvic alignment on total hip arthroplasty. J Arthroplasty 2018;33(5):1606-16. https://doi.org/10.1016/j.arth.2017.11.021

7. Esposito CI, Gladnick BP, Lee YY, Lyman S, Wright TM, Mayman DJ, et al. Cup position alone does not predict risk of dislocation after hip arthroplasty. J Arthroplasty 2015;30:109-13. https://doi.org/10.1016/j.arth.2014.07.009

8. Lazennec JY, Clark IC, Folinais D, Tahar IN, Pour AE. What is the impact of a spinal fusion on acetabular implant orientation in functional standing and sitting positions? J Arthroplasty 2017;32:3184-90. https://doi.org/10.1016/j.arth.2017.04.051

9. Lazennec JY, Brusson A, Rousseau MA. Hip-spine relations and sagittal balance clinical consequences. Eur Spine J 2011;20(Suppl 5):S686-S698. https://doi.org/10.1007/s00586-011-1937-9

10. Lazennec JY, Rousseau MS, Rangel A, Gorin M, Belicourt C, Brusson A, et al. Pelvis and total hip arthroplasty acetabular component orientations in sitting and standing positions: measurements reproductibility with EOS imaging system versus conventional radiographies. Orthop Traumatol Surg Res 2011;97(4):373-80. https://doi.org/10.1016/j.otsr.2011.02.006

11. Lazennec JY, Ramaré S, Arafat N, Laudet CG, Gorin M, Roger B, et al. Sagittal alignment in lumbosacral fusion: relations between radiological parameters and pain. Eur Spine J 2000;9(1):47-55. https://doi.org/10.1007/s005860050008

12. Lazennec JY, Boyer P, Gorin M, Catonné Y, Rousseau MA. Acetabular anteversion with CT in supine, simulated standing, and sitting positions in a THA patient population. Clin Orthop Relat Res 2011;469(4):1103-9. https://doi.org/10.1007/s11999-010-1732-7

13. Lafage R, Ferrero E, Henry JK, Challier V, Diebo B, Liabaud B, et al. Validation of a new computer-assisted tool to measure spino-pelvic parameters. Spine J 2015;15(12):2493-502. https://doi.org/10.1016/j.spinee.2015.08.067

14. Stefl M, Lundergan W, Heckmann N, McKnight B, Ike H, Murgai R, et al. Spinopelvic mobility and acetabular component position for total hip arthroplasty. Bone Joint J 2017;99-B(1 Suppl A):37e45. https://doi.org/10.1302/0301-620X.99B1.BJJ-2016-0415.R1

15. Ike H, Dorr LD, Trasolini N, Stefl M, McKnight B, Heckmann N. Spine-pelvis-hip relationship in the functioning of a total hip replacement. J Bone Joint Surg Am 2018;100(18):1606-15. https://doi.org/10.2106/JBJS.17.00403

16. Lum ZC, Coury JG, Cohen JL, Dorr LD. The current knowledge on spinopelvic mobility. J Arthroplasty 2018;33:291-6. https://doi.org/10.1016/j.arth.2017.08.013

17. Kanawade V, Dorr LD, Wan Z. Predictability of acetabular component angular change with postural shift from standing to sitting position. J Bone Joint Surg Am 2014;96:978-86. https://doi.org/10.2106/JBJS.M.00765 
18. Malkani AL, Garber AT, Ong KL, Dimar JR, Baykal D, Glassman SD, et al. Total hip arthroplasty in patients with previous lumbar fusion surgery: are there more dislocations and revisions? J Arthroplasty 2018;33(4):1189-93. https://doi.org/10.1016/j.arth.2017.10.041

19. Patel PD, Potts A, Froimson MI. The dislocating hip arthroplasty: prevention and treatment. J Arthroplasty 2007;22(4 Suppl 1):86-90. https://doi.org/10.1016/j.arth.2006.12.111

20. Delaunay C, Hamadouche M, Girard J, Duhamel A, Group S. What are the causes for failures of primary hip arthroplasties in France? Clin Orthop Relat Res 2013;471(12):3863-9. https://doi.org/10.1007/s11999-013-2935-5

21. Bedard NA, Martin CT, Slaven SE, Pugely AJ, Mendoza-Lattes SA, Callaghan JJ. Abnormally high dislocation rates of total hip arthroplasty after spinal deformity surgery. J Arthroplasty 2016;31:2884-5. https://doi.org/10.1016/j.arth.2016.07.049

22. Perfetti DC, Schwarzkopf R, Buckland AJ, Paulino CB, Vigdorchik JM. Prosthetic dislocation and revision after primary total hip arthroplasty in lumbar fusion patients: a propensity score matched-pair analysis. J Arthroplasty 2017;32(5):1635-40.e1. https://doi.org/10.1016/j.arth.2016.11.029

23. Buckland AJ, Puvanesarajah V, Vigdorchik J, Schwarzkopf R, Jain A, Klineberg EO, et al. Dislocation of a primary total hip arthroplasty is more common in patients with a lumbar spinal fusion. Bone Joint J 2017;99-B(5):585-91. https://doi.org/10.1302/0301-620X.99B5.BJJ-2016-0657.R1

24. Dorr LD, Wan Z. Causes of and treatment protocol for instability of total hip replacement. Clin Orthop Relat Res 1998;355:144-51. https://doi.org/10.1097/00003086-199810000-00015

25. Dorr LD, Wolf AW, Chandler R, Conaty JP. Classification and treatment of dislocations of total hip arthroplasty. Clin Orthop Relat Res 1983;173:151-8. https://doi.org/10.1097/00003086-198303000-00019 